\title{
The Research of Data Security Mechanism Based on Cloud Computing
}

\author{
Changyou Guo ${ }^{1,2}$ and Xuefeng Zheng ${ }^{1 ; 2}$ \\ ${ }^{1}$ School of Computer and Communication Engineering, \\ University of Science and Technology Beijing, Beijing, China \\ ${ }^{2}$ Beijing Key Laboratory of Knowledge Engineering for Materials Science \\ Beijing, China \\ guochangyouustb@139.com
}

\begin{abstract}
With the wide use of cloud computing services, users require higher and higher security. So the safety of cloud computing is the first consideration of users to choose. In the development of cloud computing, the application proportion of virtualization gradually increase, the scope and depth of the safety gradually expand. The related concepts of cloud computing and development situation are introduced in this paper. Not only the key technologies of cloud computing security are analyzed, but also a cloud security framework is put forward combined with the current security problems needed to resolve in cloud computing. We analyze and compare the present research results of security model and mechanism in the cloud. At last, we propose a security mechanism based on cloud computing.
\end{abstract}

Keywords: Cloud Computing, Cloud security, data security, Security services, virtualization security

\section{Introduction}

Cloud computing is a new concept in recent years, and a newly computing framework is proposed. Cloud computing is the development of distributed computing, parallel computing and grid computing [1]. The goal of cloud computing is to simplify the computing and storage for like public water and electricity, the user can be convenient to use these resources only could be connected to network, and to pay by the volume that they used. Cloud computing is usually have a distributed infrastructure, and can carry on real-time monitoring of the distributed system, in order to achieve the efficient usage of it [2]. The computing make computers act on the cloud and the computer makes parallel computing technology into people's life [3]. Users service themselves relying on some internet information resources which lie on some nodes, such as computing resources, software resources, data resources and management resources. This service framework emphasize the demand driven, user dominant, on-demand services, no centralized control and users don't care where the server. The parallel computing and virtualization technology has become the core support technology after the concept of cloud computing was put forward.

There are existing two means of cloud computing [4]: one aspect is describes the infrastructure, which used to construct applications and the role equivalents to the PC operating system: the other aspect is describes cloud computing applications based on the infrastructure. The different from grid computing is that cloud computing have a wider meaning about the computing platform, and can support the application of the grid. The 
existing implementation of cloud computing reflects characteristics of the following three aspects:

1) The hardware infrastructure established on large-scale and cheap server cluster.

2) The collaborative development between application and the underlying service, maximize the use of resources.

3) Through multiple redundancies between cheap servers, achieved high availability of software.

IT resource service is the most important external characteristics of cloud computing. At present, Amazon, Google, IBM, Microsoft, Sun and other IT companies establish and provide all kinds of cloud computing services. According to the national institute of standards and technology (NIST).

The current cloud computing services can be divided into three levels, respectively is[5]: (1) the infrastructure as a service (IaaS), such as the Amazon elastic compute cloud (or EC2), IBM blue cloud [6] and Sun's cloud infrastructure platform (IaaS) [7], etc., (2) the platform as a service (PaaS), such as Google AppEngine [8] and Microsoft's Azure, etc., (3) software as a service (SaaS), such as Salesforce customer relationship management (CRM) services, etc.

\section{A. Infrastructure as a Service (IaaS)}

The First layer is the IaaS. The capability provided to the customer of IaaS is raw storage space, computing, or network resources with which the customer can run and execute an operating system, applications, or any software that they choose. The cloud customer is not able to control the distribution of the software to a specific hardware platform or change parameters of the underlying infrastructure, but the customer can manage the software deployed.

\section{B. Platform as a Service (PaaS)}

Second layer is the PaaS. In the case of PaaS, the cloud provider not only provides the hardware, but they also provide a toolkit and a number of supported programming languages to build higher level services (i.e., software applications that are made available as part of a specific platform). The users of PaaS are typically software developers who host their applications on the platform and provide these applications to the end-users.

\section{Software as a Service (SaaS)}

Third and last layer is the SaaS. The SaaS customer is an end-user of complete applications running on a cloud infrastructure and offered on a platform on-demand. The applications are typically accessible through a thin client interface, such as a web browser. The customer does not control either the underlying infrastructure or platform, other than application parameters for specific user settings.

According to various ways of implementation adopt on service model, its support relation with physical infrastructure as shown in Figure 1 [9]. 


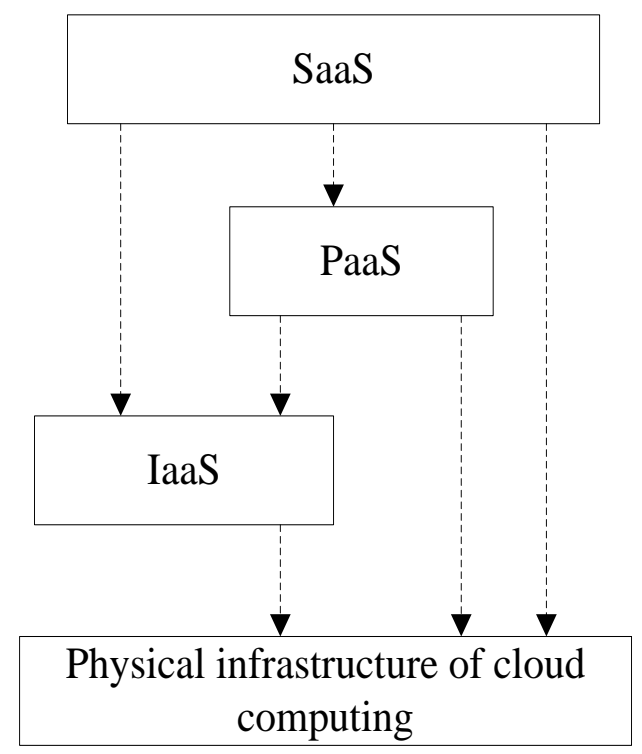

Figure 1. The Service Delivery Model for Cloud Computing

This paper is organized as: literature review is presented in 2, cloud challenges and key technologies are presented in 3, a new cloud security framework are provided in 4, and the paper is concluded in 5 .

\section{Literature Review}

A system that could capture information on the cloud is proposed, through this information the type of security could be identified and this result will make users know their information is securing not threats. The system's implementation is based on the relatively small clouds, so the extending of this framework is required [10].

Based on the security properties between applications and the analysis of data management a data-centric framework of cloud security is proposed. The advantage of this framework is showed using Declarative Secure Distributed Systems platform. Several directions are the further development of this paper [11].

According to the features of the major security and privacy issues in the cloud computing, four effective methods are proposed. These methods could deal with one or two aspects such as the security and privacy issues [12].

Based on cloud computing and cloud operating systems the unique and shared security issues between cloud and traditional computing are discussed in this paper. In addition a method that allows the user to select security level and a list of security items is proposed [13].

Some feasible and available solution used to solve reliability, availability and security issues are proposed. Such as access control and incident countermeasure and response. Partitioning, migration and workload analysis and allocation are the major challenges [14].

Based on the bilinear pairing techniques a new secure provenance framework is proposed. Anonymous authentication, provenance track and information confidentiality are complete in proposed scheme. And the secure of this scheme are demonstrated [15].

A concise of all analysis of the challenges faced by cloud computing is discussed in this paper, such as authentication, dynamic virtualization management, secure hose and guest 
operation system, privacy protection and so on. Some solutions available to these challenges are also provided [16].

In order to pinpoint the challenges and issues of cloud computing, some problems are listed in this paper. Such as computing paradigms, adoption perspective and interoperability issue [17].

The privacy, security, reliability, access and regulation of cloud computing are discussed. Not only nature and potential issues are explored but also the policy issues are showed [18].

\section{Challenges and Key Technology for Cloud Computing Security}

The main concern of cloud computing is the safety issue according to latest survey of IDC. Therefore, the security of user data will be the key factor decided cloud computing for enterprise applications [19]. Survey of Gartner in 2009 showed that more than $70 \%$ of the respondents in the actual deployment of cloud computing is security and privacy issues [20]. Cloud computing characterized by dynamic services as the main technical, flexible "service contract" as the core business characteristics, is undergoing significant changes. This change has brought huge impact for the information security field. At least, the key technology should be solved to realize the cloud computing security, construction standards and regulations as well as the national supervision and management system of multiple levels of challenge [21].

The main challenges of cloud computing security are refers as these aspects, the cloud security technology framework that the main target is the establishment of data security and privacy protection, the cloud computing safety standard and assessment system which core is established a safety target validation and safety service level evaluation. And the last one is the security regulatory system that could be controllable.

According to Gartner's survey report [22] that was showed by Figure 2, the security, reusability and performance are the three aspects that the users are most attentions.

\section{Q: Rate the benefits commonly ascribed to the 'cloud'/on-demand model}

(Scale: $1=$ Not at as mportant $\$=$ Very Important)

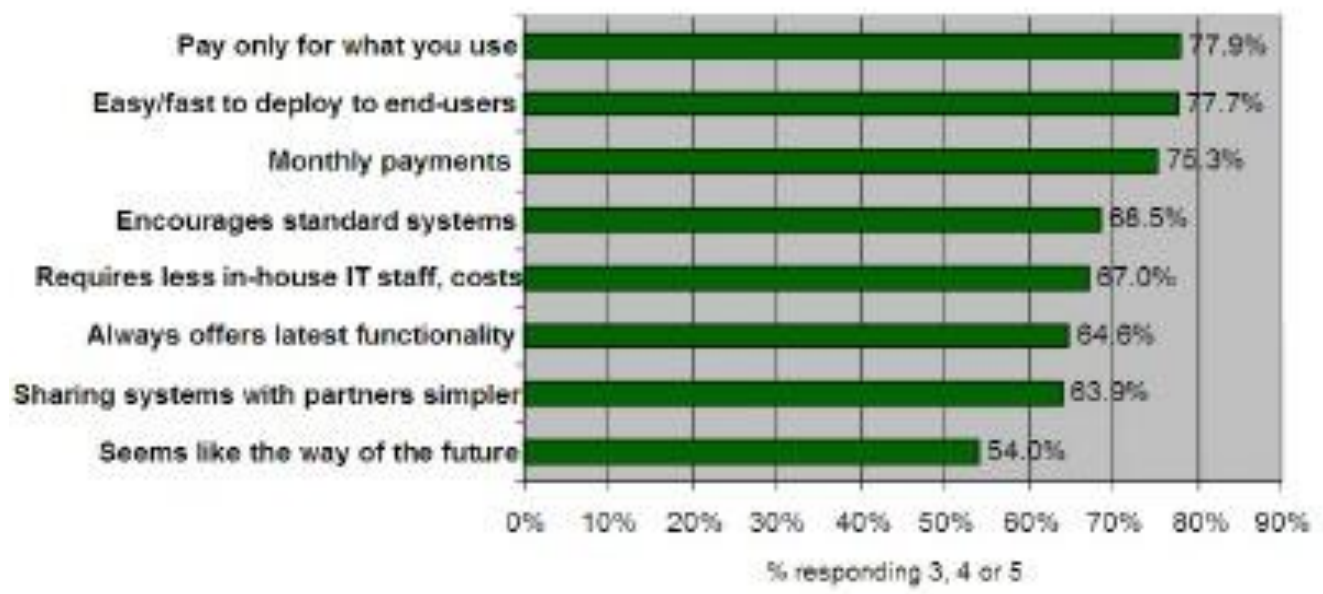

Source: IDC Enisrpries Pensi, 2000, n = 203

Figure 2. The Challenges for Cloud Computing

The key technology [21] of cloud computing security mainly includes:

1) trusted access control; 
2) lack of standards;

3) cipher text retrieval and processing;

4) data exists and reusability;

5) data privacy;

6) virtual security technology;

7) Denial of service.

With the further development of virtualization technology and further promotion of cloud computing technology, more and more users will use a third party cloud computing services. And, when users transfer the important data to cloud computing center, internal attacks may appear more on user data in the center of cloud computing in the future. There have been more and more foreign standards organization started to develop cloud computing and safety standards, in order to enhance the interoperability and security, reduce duplication of investment or reinvent, some organizations such as ITU-TSG17 team [23] launched the cloud computing standard work. In addition, some specially group, such as cloud computing security alliance also has made certain progress in cloud computing security standardization.

In the domestic IT industry, all kinds of cloud computing security products and solutions appear. For example, Sun Microsystems released open source cloud computing security tools for Amazon's EC2, S3, and virtual private cloud platform to provide security protection. Tools including OpenSolaris VPC gateway software, can help customers quickly and easily create a gateway to Amazon virtual private cloud multiple security communication channel; VMIs that designed for Amazon EC2, including non-executable stack, the encrypted exchange and enabled by default audit, etc., Cloud security boxes, using of class Amazon S3 interface, automatically compression, encryption, and split the content, simplifying the encrypted content in the cloud management.

\section{Proposed Work}

The priority problem of cloud computing security needed to solve are these: establish a comprehensive cloud computing security framework according threats, and actively carry out various key technology research. The important research to analysis and solve of cloud computing is computing mode of cloud computing services, dynamic virtualization management way and many tenants share the operating mode that threat the data security and privacy protection. The security problems caused by computing framework and by dynamic virtualization management mode of security problems, multi-level service mode of security in cloud computing, etc. Due to the lack of security key technology support, the current cloud platform service adopted by most business to avoid the above problems. But in the long run, the security and privacy protection of user data needs is the core problem that is unable to avoid.

In fact, the above problem is not lack of technology, such as data outsourcing and service outsourcing security, trusted computing environment, computation of virtual machine safe and secret homomorphism various technique for many years to be the concern of the academia. The key to realize the above technology in the practical application of cloud computing environment, supporting the key technology of the future cloud computing security system, and ultimately provide cloud services that has the security. The important security objectives of cloud users are the data security and privacy protection services.

A cloud security framework is put forward through the research on data security in cloud computing security mechanism. As shown in figure 3 :

In cloud computing environment, the traditional security protection framework cannot solve all problems, so a new security protection system is needed. 
Figure 3 mainly includes physical security, virtual network security, cloud operating system security, virtual cluster security, SaaS/PaaS/IaaS security, data security, safety management and safety operational and so on. In fact, cloud computing is introduced into the virtualization technology, and changed the service way, but did not overthrow the traditional safe mode.

We can see from Figure 3 that due to the introduction of virtualization technology, virtualization security protection measures needed to be included. But in the basic level, the mature traditional security technology needs to provide security protection.

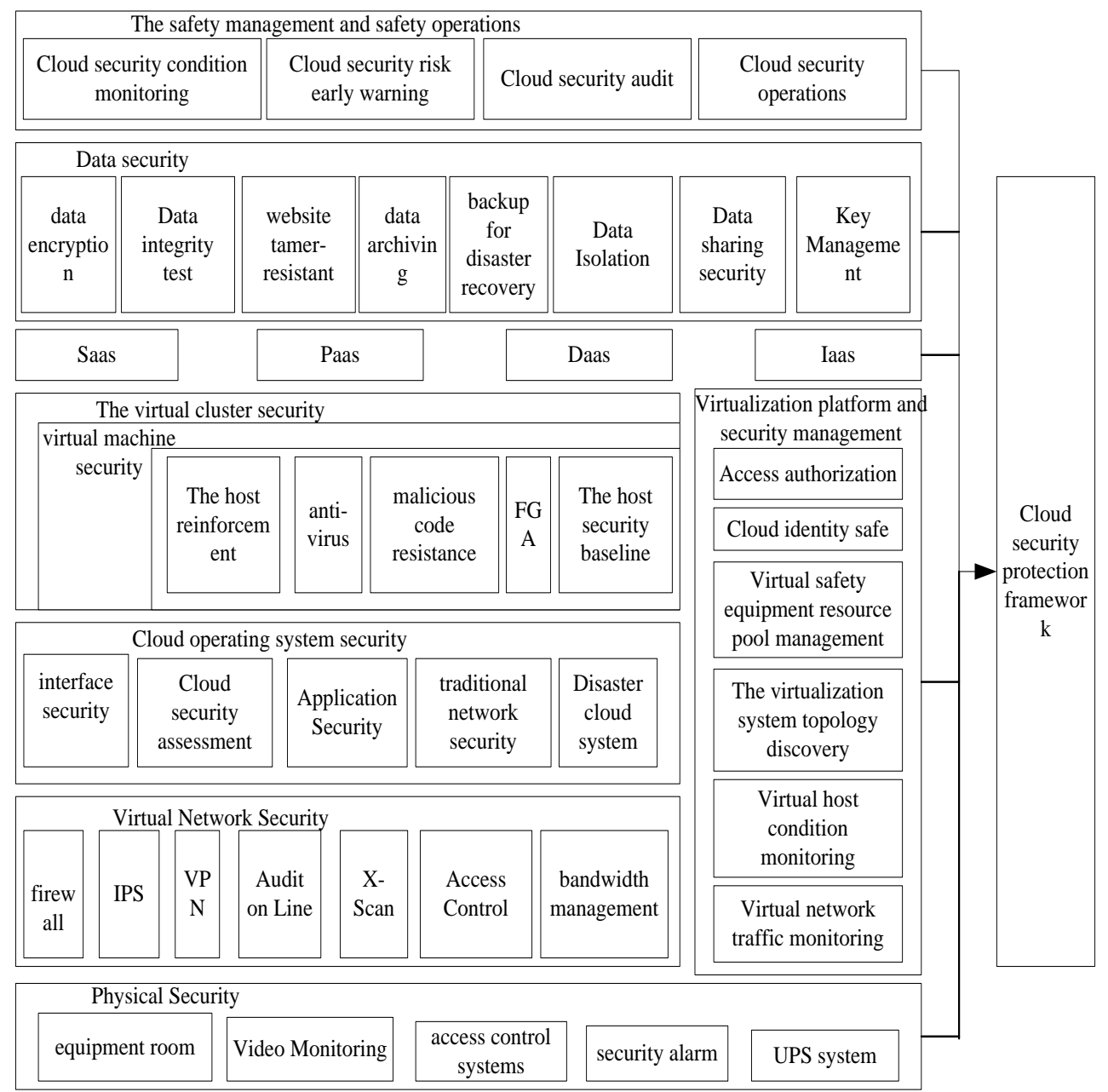

Figure 3. The Proposed Cloud Security Framework

Cloud computing security and traditional security have the same security objectives in security target, system resource type, security technology. But the cloud has its unique security issues, mainly includes: virtualization security issues and some safety problems related to rent service mode. We can put the cloud security as a superset of traditional security, or in other words, the cloud security is the succession and development of traditional security in cloud computing environment. Thus a basic understanding of cloud computing can be deduced: the cloud computing framework is a revolutionary: virtualization security, data security and privacy protection is the key and difficult point problems in cloud 
computing. Cloud security will be get development based on the traditional security technology.

\section{Conclusions}

The data security issues under cloud computing environment have become a key consideration for users to choose it. The challenge is also unprecedented. A cloud security framework is proposed through the research on data security in cloud computing security mechanism. Cloud computing security refers some problems, such as technology, standardization, business regulations, laws and regulations, and many other fields. So, only from a technical perspective to solve safety problems of cloud computing is not enough, need the joint efforts of information security in academia, industrial circle, enterprise, government and related departments.

\section{ACKNOWLEDGMENTS}

This work was supported by the Natural Science Foundation of China (No.61163025) and the Project of Beijing Key Laboratory of Knowledge Engineering for Materials Science (No.Z121101002812005).

\section{References}

[1] B. Greg, M. Padma, Q. Dennis, et al., "Cloud Computing", [EB/OL] http://www.Cloud computing-china.cn.

[2] P. Wang, "The key technology of cloud computing and application", Beijing: Posts and Telecom Press, (2010).

[3] K. Chen and W. M. Zheng, "Cloud computing: System instances and current research", Journal of Software, vol. 20, no. 5, (2009), pp. 1337-1348.

[4] S. Ullah and Z. Xuefeng, "Cloud Computing Research Challenges", ArXiv preprint arXiv:1304.3203, (2013).

[5] “IBM LanYun solution”, http://www-900.ibm.com/ibm/ideasfromibm/cn /cloud/solutions/index.shtml.

[6] "SUN Cloud computing architecture is introduced, the white paper", http://developers.sun.com.cn/blog/functionalca/resource/sun_353cloudcomputing_chinese.pdf.

[7] L. A. Barroso, J. Dean and U. Holzle, "Web search for a planet: The Google cluster architecture", IEEE Micro, vol. 23, no. 2, (2003), pp. 22-28.

[8] M. Almorsy, J. Grundy and I. Müller, "An analysis of the cloud computing security problem", Proceedings of APSEC 2010 Cloud Workshop, Sydney, Australia, (2010) November 30.

[9] L. Q Sumter, "Cloud Computing: Security Risk," 48th ACM Southeast Regional Conference, (2010).

[10] W. Zhou, et al., "Towards a Data Centric View of Cloud Security," 2nd International Workshop on Cloud Data Management, (2010), pp. 25-32.

[11] Z. Wang, "Security and Privacy Issues within the Cloud Computing,"2011 International Conference on Computational and Information Sciences, (2011), pp. 175-178.

[12] J. C. Roberts II and W. Alhamdani, "Who Can You Trust in the Cloud?: a Review of Security Issues within Cloud Computing", ACM International Security Curriculum Development Conference, (2011), pp. 15-19.

[13] F. Sabahi, "Cloud Computing Security Threats and Responses," 3rd IEEE International Conference on Communication Software and Networks, (2011), pp. 245-249.

[14] R. Liu, et al., "Secure Provenance: The Essential Bread and Butter of Data Forensic in Cloud Computing", 5th ACM Symposium on Information, Computer and Communication Security, (2010), pp. 282-292.

[15] S. Ullah, Z. Xuefeng, Z. Feng, et al., "Tcloud: Challenges And Best Practices For Cloud Computing", arXiv preprint arXiv:1304.3134, (2013).

[16] K. Popvic and Z. Hocenski, "Cloud Security Issues and Challenges," 33rd International Convention on Information and Communication Technology, Electronics and Microelectronics, (2010), pp. 344-349.

[17] P. T. Jaeger, J. Lin and J. M. Grimes, "Cloud computing and information policy: Computing in a policy cloud?", Journal of Information Technology \& Politics, vol. 5, no. 3, (2008), pp. 269-283.

[18] R. John and R. James, "Cloud Computing: Implementation, Management, and Security", 2009 March.

[19] T. Z. Xia and Z. Li, "Research on Cloud Computing Based on Deep Analysis to Typical Platforms", cloudcom, beijing, China, (2009), pp. 601-608. 
International Journal of Security and Its Applications

Vol. 9, No. 3 (2015)

[20] D. G. Feng, M. Zhang, Y. Zhang and Z. Xu, "Study on cloud computing security. Journal of Software, vol. 22, no. 1, (2011), pp. 71-83.

[21] http://blogs.idc.com/ie/?p=730.

[22] ITU. http://www.itu.int/en/pages/default.aspx.

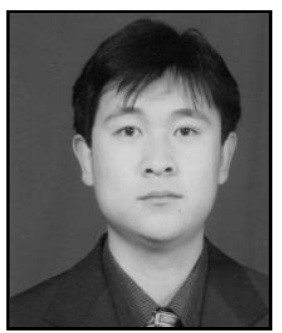

Guo Changyou, was born in 1976 , is currently a $\mathrm{PhD}$ candidate at the School of Computer and Communication Engineering, University of Science and Technology, Beijing. His research interest includes Access Control, Network Security, Information Security and Cloud Computing Security.

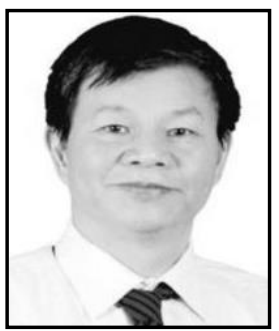

Zheng Xuefeng, was born in 1951, is professor and doctoral supervisor in the School of Computer and Communication Engineering, University of Science and Technology Beijing. His research interest includes Computer Control Systems Development, Computer System Security Analysis, Network Security, Information Security and Distributed Systems Security. He is the senior member of the computer society. 